\title{
Impact of Admission Hyperglycemia on Stroke-Associated Pneumoniain Acute Cerebral Hemorrhage: A Retrospective Observational Study
}

\author{
Tian-Hua Ren1, Biao Yuan², Tie-Cheng Yang1, Jian-Guo Li1, Niu Chi', Qian-Wei Ren³, \\ Hong-Mei Shi ${ }^{4}$, Dong-Ming Yu', Kai Shan', Li-Xia Li' ${ }^{1}$ Xiao-Yan Dong1 \\ ${ }^{1}$ Department of Emergency of Beijing Tiantan Hospital, Capital Medical University, Beijing, China \\ ${ }^{2}$ Department of Vascular Surgery, Beijing Chaoyan Hospital, Capital Medical University, Beijing, China \\ ${ }^{3}$ Department of Clinical Medicine, Tianjin Medical University, Tianjin, China \\ ${ }^{4}$ Chongwen District Centers for Disease Control and Prevention, Beijing, China \\ Email: renth1970@163.com
}

Received 10 June 2015; accepted 20 July 2015; published 23 July 2015

Copyright (C) 2015 by authors and Scientific Research Publishing Inc.

This work is licensed under the Creative Commons Attribution International License (CC BY). http://creativecommons.org/licenses/by/4.0/

(c) (i) Open Access

\section{Abstract}

Background: Hyperglycemia is always seen amongst acute intra-cerebral hemorrhage (ICH) and usually has been reported in literature and studied in relation to mortality and poor recovery. However, literature specific to stroke-associated pneumonia (SAP) on this topic is very small. Further, how to differentiate the predictive value of hyperglycemia with and without abnormal HbA1C in such patients is still a matter of debate and no universal consensus. We evaluated hyperglycemia as a marker for SAP in patients with ICH to assess its usefulness as a potential predictor. Materials and methods: Clinical characteristics for a sample of 551 patients with acute ICH were collected from the Beijing Tiantan Hospital of Capital Medical University, Beijing, China. Possible associated risk factors of SAP were reviewed. Hyperglycemia and HbA1C on admission were the main hypothetic predictor, SAP occurring within the first 7 days is the primary outcome. Results: The cohort study includes 551 hospitalized patients. The prevalence of hyperglycemia was $52.5 \%$ and SAP occurred in $147(26.7 \%)$. The incidence of SAP was higher in the group with hyperglycemia than those without hyperglycemia $(37.7 \%$ versus $14.5 \%, p<0.01)$. Multivariate logistic regression analyses showed the SAP was significantly associated with hyperglycemia (OR, $2.48 ; 95 \%$ CI, 2.37 - 5.72), in the subgroup analysis we found hyperglycemia with normal HgbA1C level $(<6.5)$ had been shown to be significantly associated with increased incidence of SAP (OR, 3.86; 95\%CI, 2.98 - 6.72), however, hyperglycemia with high HgbA1C level (>6.5) (OR, 1.57; 95\%CI, 0.81 - 3.23) had not been shown to be associated with SAP. Conclusions: In this hospital-based co- 
hort of patients presenting with acute intra-hemorrhage, hyperglycemia on admission was associated significantly with SAP. The association was stronger for hyperglycemia with normal HgbA1C than for hyperglycemia with high HgbA1C. Hyperglycemia with normal HgbA1C might be a more sensitive predictor of early acute complication, such as SAP.

\section{Keywords}

\section{Acute Intracerebral Hemorrhage (ICH), Stroke-Associated Pneumonia (SAP), Hyperglycemia, Hemoglobin (Hb)A1C}

\section{Introduction}

Post-stroke infection is the most common serious medical complication and is a significant contributor to morbidity and mortality. Of post stroke infections pneumonia frequently complicates stroke and can have a major impact on outcome. A recent meta-analysis of post-stroke infection in a large group of patients with ischemic and hemorrhagic stroke found a $10 \%$ infection rate [1]. In these infection, the incidence of stroke-associated pneumonia (SAP) may as high as $40 \%$, it occurs most frequently during the first week after stroke and especially the first 3 days after stroke onset [1] [2]. SAP independently increases in-hospital mortality 2 - 6 times [3]-[6]. There is an increasing body of literature on the importance of SAP after acute ischemic stroke, but information on SAP after intra-cerebral hemorrhage (ICH) is limited [7]. To identify patients at greatest risk in the acute phase of ICH is of fundamental importance in approaching prevention and treatment. Other studies have identified risk factors for SAP after ICH, broadly including age, volume of hemorrhage, premorbid dependence, and enteral feeding, comorbidities, mechanical ventilation and stroke severity or stroke-associated deficits such as somnolence and dysphagia [8]-[10]. However, up to now there are only few studies of the role of the initial blood glucose levels in promoting SAP in patients with ICH have been reported. Hyperglycemia on admission is a well-known predictor of poor outcome in patients with ICH [11] [12]. Recently, there are few small studies have examined the relationship between blood glucose levels and post-stroke infection [13] [14]. No larger studies have examined the incidence of SAP or their impact on outcome in a pure ICH cohort. Given the lack of robust data on SAP after ICH, we seek to determine the relationship between the initial Hyperglycemia and SAP in acute phase of patients with ICH.

\section{Patients and Methods}

This study was conducted at the Beijing Tiantan Hospital of Capital Medical University, Beijing, China, with approval of the hospital's institutional review board. Data collected from June 2008 to December 2009 for patients with ICH were retrospectively reviewed as we before reported [15]. ICH was defined as a neurological deficit with confirmation of the brain hemorrhage by computed tomography (CT). Two independent physicians reviewed all the available records (medical and nursing notes) of ICH. All cases met the following eligibility criteria were listed in the following: (1) age $\geq 18$ years; (2) hospitalized with a primary diagnosis of acute spontaneousintrcranialhemorrhage stroke according to World Health Organization criteria [16]; (3) stroke confirmed by CT. Patients with any of the following were excluded: hemorrhage secondary to brain tumors, trauma, hemorrhagic transformation of cerebral infarct, or aneurismal or vascular malformation rupture. We also excluded admission with incomplete medical data and those $<7$ days in duration. Demographic and clinical data included patient's age, sex, medical history, initial Glasgow Coma Scale (GCS) score, initial laboratories results, head CT findings and in-hospital treatments for ICH (i.e, external ventricular drainplacement or surgical hematoma evacuation). Neuroradiological findings were determined in theinitial CT scan. These were classified according to the sites of ICH (basal ganglia, thalamic, lobar, pontine, or cerebellar), volume of hematoma and intra-ventricular extension after intra-ventricularhemorrhage (IVH). For the study, the following risk factors for SAP that have been identified were analyzed, such as age, male sex, stroke severity (GCS, NIHH), dysphagia, mechanical ventilation, diabetes mellitus, chronic pulmonary disease, history of pneumonia, the use of sedative drugs. Serum blood glucose and hemoglobin (HgB)A1C values obtained basically in the emergency department at initial presentation were evaluated for each patient as clinical characteristicst. We defined hyperglycemia as more than 
1 random blood glucose level $\geq 11.0 \mathrm{mmol} / \mathrm{L}$ [17]. Patients with any missing these blood glucose parameters were excluded from this study. The blood glucose management including extra measurements and control depended on attending physician decision. In this study, the primary outcome measure was clinician diagnosis of SAP based on initiation of antibiotic therapy occurring within the first 7 days after admission as recorded by medical and nursing notes. SAP was diagnosed by treating physician according to the Centers for Disease Control and Prevention criteria for hospital-acquired pneumonia [18]. In our analyses, data are presented as median values (interquartile range) or number (\%). Univariate analyses were undertaken to determine the clinical factors associated with SAP in the derivation cohort. Univariable hypothesis tests used were based on $X^{2}$ tests, and independent sample $t$ test. A risk for SAP was derived by fitting a multivariable logistic regression model to the derivation data. Initial variables were selected based on the results of the univariate analyses, clinical experience, and previously published literature on Sap risk factors. A p value of $<0.05$ was considered significant.

\section{Results}

\subsection{Patient Admission Baseline Characteristics}

We enrolled 808 individuals into this study between June 2008 and December 2009, of which 257 patients were excluded from this analysis (counts not mutually exclusive): 149 by design (76 with death, withdrawal care, or discharge to hospice within 72 hours; 73 with recent infection); and 108 because of missing data; (47 with incomplete computed tomographic data; 61 with unavailable clinical data).Patients excluded because of missing data did not differ from included patients with respect to known predictors of ICH outcome (ICH size and location, intraventricular hemorrhage, and presenting GCS). Table 1 shows the baseline characteristics and the initial laboratory data of the included patients according to diabetic mellitus and glycemia on admission. 289 patients (52.5\%, $p=0.214)$ had hyperglycemia. Admission factors associated with hyperglycemia in univariate analyses $(p<0.05)$ included admission GCS, ICH volume, EVD (68.2\% versus $14.5 \% ; p<0.01)$, IVH $(21.8 \%$ versus $8.4 \% ; p<0.01$ ). History of pneumonia (21.1\% versus $14.1 \%$; $p<0.01$ ), WBC (8.9 versus $5.2 ; p<0.01$ ).

\subsection{Prevalence and Predictors of SAP}

SAP occurred in 147 of the 551 patients (26.7\%). Admission characteristics are reported in Table 2. Admission factors associated with SAP in univariate analyses $(p<0.05)$ included admission GCS (7 versus $9 ; p<0.01$ ), IVC (21.1\% versus $13.4 \% ; p<0.01$ ), dysphagia ( $53.7 \%$ versus $29.2 \% ; p<0.01)$, mechanical ventilation $(61.9 \%$ versus $21.5 \% p<0.01$;), history of pneumonia ( $8.2 \%$ versus $2.7 \% ; p<0.01)$, sedative use $(72.8 \%$ versus $52.2 \%$ $p<0.01$;), hyperglycemia ( $74.1 \%$ versus $44.6 \% p<0.01)$. In multivariate models including both admission and hospital-related risk factors, SAP were associated with mechanical ventilation (OR, 4.76; 95\% CI, 3.13 - 6.74), dysphagia (OR, 3.16; 95\% CI, 2.32 - 5.19), history of pneumonia (OR, 7.12; 95\% CI, 4.42 - 11.35), sedative use (OR, 3.61; 95\% CI, 2.83 - 8.64), hyperglycemia (OR, 2.48; 95\%CI, 2.37 - 5.72), whereas GCS, IVH, HbA1C did not reach statistical significance.

\subsection{HbA1C Subgroup and Sensitivity Analysis}

In order to analysis the sensitivity of hyperglycemia to access SAP, we divided SAP group (147 cases) into two subgroups by the HbA1C burden ( $>$ or $<6.5 \%$ ). Inmultivariate logistic regression models included all variables in Table 2. We found a significant association for hyperglycemia (OR, 3.86; 95\%CI, 2.98 - 6.72) with SAP in HbA1C > 6.5 subgroup; but not for hyperglycemia (OR, 1.57; 95\%CI, 0.81 - 3.23) in HbA1C > 6.5 subgroup; see Table 3.

\section{Discussion}

SAP is a common medical complication after acute stroke. Although the treatment of acute stroke has improved, the impact of SAP on mortality may have changed little in recent years [19]. Prediction of patients at greatest risk of SAP could be of major importance in individualized monitoring and prognostication, along with risk stratification in clinical trials of preventative therapies (eg, antibiotics). In this study several risk factors for SAP have been identified. We confirmed that SAP within the first 7 days after admission was significantly associated with mechanical ventilation (OR, 4.76; 95\% CI, 3.13 - 6.74), dysphagia (OR, 3.16; 95\% CI, 2.32 - 5.19), history of pneumonia (OR, 7.12; 95\% CI, 4.42 - 11.35), sedative use (OR, 3.61; 95\% CI, 2.83 - 8.64). We also confirmed 
Table 1. Baseline characteristics of the study population stratified according to diabetic mellitus and glycaemia on admission.

\begin{tabular}{|c|c|c|c|c|c|c|c|}
\hline & Patients & $\begin{array}{c}\text { Patients } \\
\text { with Diabetes } \\
\text { Mellitus, }\end{array}$ & $\begin{array}{c}\text { Patients } \\
\text { without Diabetes } \\
\text { Mellitus, }\end{array}$ & p-Value & $\begin{array}{l}\text { Patients with } \\
\text { Hyperglycemia } \\
\text { on Admission }\end{array}$ & $\begin{array}{l}\text { Patients without } \\
\text { Hyperglycemia } \\
\text { on Admission }\end{array}$ & P-Value \\
\hline No. patients & 551 & $113(20.5 \%)$ & 438 (79.5\%) & & 289 (52.5\%) & $262(47.5 \%)$ & 0.214 \\
\hline Age, Median (range) & $56(23-88)$ & $61(34-88)$ & $54(23-71)$ & & $52(23-88)$ & $58(27-74)$ & 0.37 \\
\hline Male, NO. (\%) & $286(51.9 \%)$ & $101(52.7 \%)$ & $185(51.7 \%)$ & 0.961 & $152(52.6 \%)$ & $134(51.1 \%)$ & 0.952 \\
\hline *GCS, NO. (\%) & & & & $<0.01$ & & & $<0.01$ \\
\hline$<8$ & $231(41.9 \%)$ & $113(58.5 \%)$ & $118(33.0 \%)$ & & $167(57.8 \%)$ & $64(24.4 \%)$ & \\
\hline$>8$ & $320(58.1 \%)$ & 80 (41.5\%) & $240(67.0 \%)$ & & $122(42.2 \%)$ & $198(75.6)$ & \\
\hline \multicolumn{8}{|l|}{ *Site of ICH, NO. (\%) } \\
\hline Thalamus & $86(15.6 \%)$ & $26(13.5 \%)$ & $60(16.8 \%)$ & 0.683 & 42 (14.5\%) & $44(16.8 \%)$ & 0.732 \\
\hline Basal ganglia & $262(47.5 \%)$ & $91(47.2 \%)$ & $171(47.8 \%)$ & 0.954 & $148(51.2 \%)$ & $114(43.5 \%)$ & 0.747 \\
\hline Lobar & $107(19.4 \%)$ & 28 (14.5\%) & $79(22.1 \%)$ & 0.031 & 48 (16.6\%) & 59 (22.5\%) & 0.042 \\
\hline Pons & $66(12.0 \%)$ & $36(18.7 \%)$ & $30(8.4 \%)$ & $<0.010$ & 34 (11.8\%) & 32 (12.2\%) & 0.864 \\
\hline Cerebellum & $30(5.5 \%)$ & $12(6.1 \%)$ & $18(4.9 \%)$ & 0.385 & 17 (5.9\%) & $13(5.0 \%)$ & 0.912 \\
\hline *ICH volume, NO. (\%) & & & & $<0.01$ & & & $<0.01$ \\
\hline Small $(<30 \mathrm{~mL})$ & 289 (52.5\%) & 69 (35.8\%) & 220 (61.5\%) & & $122(42.2 \%)$ & $167(63.7 \%)$ & \\
\hline Large (>30 mL) & $262(47.5 \%)$ & $124(64.2 \%)$ & $138(38.5 \%)$ & & $167(57.8 \%)$ & 95 (36.3\%) & \\
\hline EVD, NO. (\%) & $235(42.6 \%)$ & $104(53.9 \%)$ & $131(36.6 \%)$ & $<0.01$ & $197(68.2 \%)$ & 38 (14.5\%) & $<0.01$ \\
\hline IVH, NO. (\%) & 85 (15.4\%) & 52 (26.9\%) & $33(9.2 \%)$ & $<0.01$ & $63(21.8 \%)$ & $22(8.4 \%)$ & $<0.01$ \\
\hline \multicolumn{8}{|l|}{ Medical history, NO. (\%) } \\
\hline Abnormal liver function & $15(2.7 \%)$ & 7 (3.6\%) & $8(2.1 \%)$ & 0.357 & $9(3.1 \%)$ & $6(2.3 \%)$ & 0.342 \\
\hline Renal insufficiency & $26(4.7 \%)$ & $14(7.3 \%) 11$ & $12(3.4 \%)$ & 0.003 & $15(5.2 \%)$ & $11(4.2 \%)$ & 0.417 \\
\hline History of pneumonia & $98(17.8 \%)$ & 45 (23.3\%) & $54(15.1 \%)$ & $<0.01$ & $61(21.1 \%)$ & 37 (14.1\%) & $<0.01$ \\
\hline History of stroke & $103(18.7 \%)$ & $61(31.6 \%)$ & $42(11.7 \%)$ & $<0.01$ & 57 (19.7\%) & $46(17.6 \%)$ & 0.364 \\
\hline \multicolumn{8}{|l|}{ *Initial laboratories } \\
\hline WBC, median (IQR) & $6.7(3.5-21.3)$ & $5.9(3.5$ - 19.8) & $7.6(5.1-21.3)$ & 0.119 & $8.9(6.3-21.3)$ & $5.2(3.5-17.8)$ & $<0.01$ \\
\hline $\begin{array}{l}\text { Initial blood glucose } \\
\text { median (IQR) }\end{array}$ & $8.7(5.1-19)$ & $10.4(7.9-17.8)$ & $9.7(5.1$ - 19) & 0.845 & $13.2(11.6$ - 19) & $7.3(5.1-11)$ & $<0.01$ \\
\hline HbA1C, median (IQR) & $5.7(4.5-9.6)$ & $6.7(6.1-9.6)$ & $4.5(4.5-5.9)$ & $<0.01$ & $6.2(4.8-9.6)$ & $5.9(4.5-7.8)$ & 0.253 \\
\hline
\end{tabular}

p-value of comparisons between groups with and without Diabetes Mellitus, Hyperglycemia on admission. EVD, external ventricular drain; GCS, Glasgow Coma Scale; ICH, intracerebral hemorrhage; IVH, intraventricularhemorrhage; WBC, white blood cell ; HbA1C,hemoglobin A1c; INQ, interquartile range. *Measured within the first $24 \mathrm{~h}$ after admission. Chi-squared test or Fisher's exact test.

that SAP was significantly associated with hyperglycemia (OR, 2.48; 95\% CI, 2.37 - 5.72) (Table 2). Hyperglycemia was revealed to occur after intra-cerebral hemorrhage and to be related to acute stroke-associated complications and hence poorer outcome. Hyperglycemia at admission seemed to be apredictive value for poststroke infection. Two hundred eighty-nine patients with ICH were screened for hyperglycemia on admission. Our study showed that hyperglycemia was a common finding in patients presenting with SAP (74.1\%). Hyperglycemia was shown to affect initial clinical presentation and to have a role in predicting SAP. In the present study, we found the incidence of SAP was $26.7 \%$, and our results showed an independent association between 
Table 2. Prevalence of SAP and multivariable logistic regression analyses to predict SAP.

\begin{tabular}{lccc}
\hline Variable & Patients with SAP & Patients without SAP & p-Value \\
\hline No. patients & $147(26.7 \%)$ & $404(73.3)$ & 0.019 \\
Age, Median (IQR) & $65(37-88)$ & $54(23-76)$ & $<15(53.2 \%)$ \\
Male, NO. (\%) & $71(48.3 \%)$ & $9(3-15)$ & 0.113 \\
${ }^{*}$ GCS, median (IQR) & $7(3-15)$ & $28(12-47)$ & $<0.01$ \\
${ }^{*}$ ICH volume, median (IQR) & $35(15-50)$ & $54(13.4 \%)$ & 0.674 \\
${ }^{*}$ IVH, NO. (\%) & $31(21.1 \%)$ & $172(42.6 \%)$ & $<0.01$ \\
${ }^{*}$ EVD, NO. (\%) & $63(42.9 \%)$ & $118(29.2 \%)$ & $<0.01$ \\
${ }^{\Delta}$ Dysphagia, NO. (\%) & $79(53.7 \%)$ & $97(21.5 \%)$ & 0.402 \\
${ }^{\Delta}$ Mechanical ventilation, NO. (\%) & $91(61.9 \%)$ & $142(35.1 \%)$ & 0.267 \\
Diabetes Mellitus NO. (\%) & $51(34.7 \%)$ & $54(13.4 \%)$ & $<0.01$ \\
Chronic pulmonary disease NO. (\%) & $11(2.7 \%)$ & $<0.01$ \\
History of pneumonia, NO. (\%) & $23(15.6 \%)$ & $211(52.2 \%)$ & $<0.01$ \\
${ }^{\Delta}$ Sedative NO. (\%) & $12(8.2 \%)$ & $180(44.6 \%)$ & 0.534 \\
${ }^{*}$ Hyperglycemia, NO. (\%) & $5.4(4.5-7.8)$ & \\
${ }^{*}$ HbA1C, median (IQR) & $107(72.8 \%)$ & $109(74.1 \%)$ & \\
\hline
\end{tabular}

Risk factors and their association with SAP frequency within 7 days after strokeonset. Proportions are shown in brackets. $*$ Measured within the first $24 \mathrm{~h}$ after admission. ${ }^{\Delta}$ Exposed within the first 7 days after admission. Chi-squared test or Fisher'sexact test.

Table 3. Rates of stroke-associated pneumonia according to glycemic Status.

\begin{tabular}{|c|c|c|c|c|}
\hline GlycemicStatus & $\begin{array}{l}\text { Patients with SAP } \\
\qquad(\mathrm{n}=147)\end{array}$ & $\begin{array}{l}\text { Adjusted } \\
\text { odds ratio }\end{array}$ & $\begin{array}{c}\text { 95\% Confidence } \\
\text { interval }\end{array}$ & p-Value \\
\hline Hyperglycemia AbsentNO. (\%) & 38 (25.9\%) & $*$ & * & * \\
\hline Hyperglycemia Present NO. (\%) & $109(74.1 \%)$ & 2.48 & $2.37-5.72$ & $<0.001$ \\
\hline \multicolumn{5}{|l|}{ Multicategory exposure variables } \\
\hline Hyperglycemia Absent NO. (\%) & $38(25.9 \%)$ & $*$ & * & * \\
\hline HbA1C < 6.5 and Hyperglycemia NO. (\%) & $87(59.2 \%)$ & 3.86 & $(2.98-6.72)$ & $<0.001$ \\
\hline HbA1C > 6.5 and Hyperglycemia NO. (\%) & $22(15.0 \%)$ & 1.57 & $(0.81-3.23)$ & 0.279 \\
\hline
\end{tabular}

Adjust for all variables in Table 2 using multivariate logistic regression model.

hyperglycemia on admission with SAP. Being well known as hyperglycemia in relation to acute stroke is common both in patients with and in patients without a diagnosis of diabetes mellitus, and it has been suggested to worsen survival. However, recent results from several clinical studies indicate that particularly patients with acute stroke and stress hyperglycemia, but not diabetes, have increased mortality [20] [21]. On the contrary, older data by Woo et al. [22] found that patients with acute ischemic stroke and similar glucose concentrations had similar outcome regardless of whether they had diabetes or not. These results mostly focused on ischemic stroke but not on hemorrhage stroke. SAP is a common medical complication and a significant cause of mortality after acute stroke, while rates of SAP are reportedly higher in patients with ICH than ischemic stroke [23] [24]. We divided SAP group (147 cases) into two subgroups by the HbA1C burden ( $>$ or <6.5). The purpose of this study was to determine the correlation of SAP with hyperglycemia in different HgbA1C levels subgroup. In our study hyperglycemia and normal HgbA1C level $(<6.5)$ have been shown to be significantly associated with increased incidence of SAP, however, hyperglycemia and high HgbA1C level (>6.5) have not been shown to be 
associated with SAP. The presence of hyperglycemia has been demonstrated to be associated with an increased risk of infection in acute stroke [6] [7]. This might be the result of suppressing immune functions by hyperglycemia secondary to impaired phagocytosis, diminished production of oxygen radicals from neutrophils, and chemotaxis [25]. On the other hand, infection as a prominent stress condition has been known to be associated with hyperglycemia through increased level of counter-regulatory hormones (e.g. cortisol, epinephrine, glucagon), activation of the inflammatory cascade, and oxidative stress [26] [27]. In this regards, SAP account for $37.7 \%$ of all precipitating causes of hyperglycemia. A large percentage of our patients (52.5\%) had hyperglycemic on admission, but only $20.5 \%$ had diabetes mellitus diagnosed pre-stroke; thus, "stress hyperglycemia" in the absence of diabetes mellitus is very common. In this work, we found a strong relationship existed between hyperglycemia with normal HgbA1C level occurring as an acute phase complication of stroke (SAP), but not for hyperglycemia with increased HgbA1C level, which was supported by previous studies indirectly [28]-[31]. An increased HbA1c level reflects poor long term glycemic control and has specific implications for the structure and function of the vascular bed, including small and large cerebral vessels. Increased HbA1c level might be a useful marker of poor functional recovery (e.g. Disability, Bedridden). The patients with an acute stroke "stress hyperglycemia" might be a more sensitive predictor of early acute complication.

Current guidelines lack evidence-based recommendations for SAP prevention in stroke patients. Identifying swallowing impairments, implementing dietary modification, head elevation, and shortened use of mechanical ventilation have been emphasized [32] [33]. Other investigators have developed tools for identifying patients at high risk for SAP, although it is unclear how this informs patient care at present [34] [35]. Our finding showed that hyperglycemia especially hyperglycemia with normal HgbA1C level were an independent risk factor for SAP in acute phase of ICH.

Our results suggest that early-tight glycaemic control of hyperglycemia with normal HgbA1C, especially in high-risk patients such as those with dysphagia and mechanical ventilator support, could be an important part of an effective prevention strategy.

There are also some limitations in this study. First, this is a single center and retrospective study that could have selection bias. Secondly, the primary outcome measure of the present study is SAP occurring within the first 7 days after admission, the exact date of SAP occurred in 7 days after hospitalization isn't documented, but SAP occurs most frequently during the first week after stroke and especially the first 3 days after stroke onset. Furthermore, the benefits of tight glycemic control (both theoretical and actual) are not taken into account in this study and more work is needed in this area. In conclusion, hyperglycemia on admission in the non-diabetic population is associated with a higher morbidity and an increased chance of developing SAP. The association is stronger for hyperglycemia with normal HgbA1C than for hyperglycemia with high HgbA1C. Hyperglycemia with normal HgbA1C might be a more sensitive predictor of early acute complication such as SAP. Our results suggest strict glucose control should be applied to SAP in patients with hyperglycemia and normal HgbA1C.

\section{References}

[1] Westendorp, W.F., Nederkoom, P.J., Vermeij, J.D., Dijkgraaf, M.G. and van de Beek, D. (2011) Post-Stroke Infection: A Systematic Review and Meta-Analysis. BMC Neurology, 11, 110. http://dx.doi.org/10.1186/1471-2377-11-110

[2] Hoffmann, S., Malzahn, U., Harms, H., Koennecke, H.C., Berger, K., Kalic, M., Walter, G., Meisel, A., Heuschmann, P.U., and for the Berlin Stroke Register and the Stroke Register of Northwest Germany (2012) Development of a Clinical Score $\left(\mathrm{A}^{2} \mathrm{DS}^{2}\right)$ to Predict Pneumonia in Acute Ischemic Stroke. Stroke, 43, 2617-2623. http://dx.doi.org/10.1161/STROKEAHA.112.653055

[3] Finlayson, O., Kapral, M., Hall, R., Asllani, E., Selchen, D., Saposnik, G., on Behalf of the Investigators of the Registry of the Canadian Stroke Network (2011) Risk Factors, Inpatient Care, and Outcomes of Pneumonia after Ischemic stroke. Neurology, 77, 1338-1345. http://dx.doi.org/10.1212/WNL.0b013e31823152b1

[4] Wilson, R.D. (2012) Mortality and Cost of Pneumonia after Stroke for Different Risk Groups. Journal of Stroke and Cerebrovascular Diseases, 21, 61-67. http://dx.doi.org/10.1016/j.jstrokecerebrovasdis.2010.05.002

[5] Tong, X., Kuklina, E.V., Gillespie, C. and George, M.G. (2010) Medical Complications among Hospitalizations for Ischemic Stroke in the United States from 1998 to 2007. Stroke, 41, 980-986. http://dx.doi.org/10.1161/STROKEAHA.110.578674

[6] Koennecke, H.C., Belz, W., Berfelde, D., Endres, M., Fitzek, S., Hamilton, F., Kreitsch, P., Mackbert, B.M., Nabavi, D.G., Nolte, C.H., Pöhls, W., Schmehl, I., Schmitz, B., von Brevern, M., Walter, G., Heuschmann, P.U., and for the Berlin Stroke Register Investigators (2011) Factors Influencing In-Hospital Mortality and Morbidity Inpatients Treated on a Stroke Unit. Neurology, 77, 965-972. http://dx.doi.org/10.1212/WNL.0b013e31822dc795 
[7] Emsley, H.C. and Hopkins, S.J. (2008) Acute Ischaemic Stroke and Infection: Recent and Emerging Concepts. Lancet Neurology, 7, 341-353. http://dx.doi.org/10.1016/S1474-4422(08)70061-9

[8] Walter, U., Knoblich, R., Steinhagen, V., Donat, M., Beneche, R. and Kloth, A. (2007) Predictors of Pneumonia in Acute Stroke Patients Admitted to a Neurological Intensive Care Unit. Journal of Neurology, 254, 1323-1329. http://dx.doi.org/10.1007/s00415-007-0520-0

[9] Hug, A., Dalpke, A., Wieczorek, N., et al. (2009) Infarct Volume Is a Major Determiner of Post-Stroke Immune Cell Function and Susceptibility to Infection. Stroke, 40, 3226-3232. http://dx.doi.org/10.1161/STROKEAHA.109.557967

[10] Harms, H., Remnitz, P., Bohner, G., Werich, T., Klingebiel, R. and Meisel, A. (2011) Influence of Stroke Localization on Autonomic Activation, Immunodepression, and Post-Stroke Infection. Cerebrovascular Disease, 32, 552-560. http://dx.doi.org/10.1159/000331922

[11] Lee, S.H., Kim, B.J., Bae, H.J., Lee, J.S., Lee, J., Park, B.J., et al. (2010) Effects of Glucose Level on Early and LongTerm Mortality after Intracerebral haemorrhage: The Acute Brain Bleeding Analysis Study. Diabetologia, 53, 429-434. http://dx.doi.org/10.1007/s00125-009-1617-z

[12] Tan, X., He, J., Li, L., Yang, G., Liu, H., Tang, S., et al. (2014) Early Hyperglycaemia and the Early-Termdeath in Patients with Spontaneous Intracerebral Haemorrhage: A Meta-analysis. Internal Medicine Journal, 44, 254-260. http://dx.doi.org/10.1111/imj.12352

[13] Kwon, H.M., Jeong, S.W., Lee, S.H. and Yoon, B.W. (2006) The pneumonia score: a simple grading scale for prediction of pneumonia after acute stroke. American Journal of Infection Control, 34, 64-68. http://dx.doi.org/10.1016/j.ajic.2005.06.011

[14] Ji, R., Shen, H., Pan, Y., Wang, P., Liu, G., Wang, Y., et al. (2013) China National Stroke Registry Investigators. Novel risk score to predict pneumonia after acute ischemic stroke. Stroke, 44, 1303-1309. http://dx.doi.org/10.1161/STROKEAHA.111.000598

[15] Yang, T.C., Li, J.-G., Ren, T.-H., Shi, H.-M., Yu, D.-M., Shan, K., et al. (2013) Gastrointestinal Bleeding After Intracerebral Hemorrhage: A Retrospective Review of 808 Cases. American Journal of the Medical Science, 346, 279-282. http://dx.doi.org/10.1097/MAJ.0b013e318271a621

[16] Sacco, R.L., Kasner, S.E., Broderick, J.P., Caplan, L.R., Connors, J.J., Culebras, A., et al. (2013) An Updated Definition of Stroke for the 21st Century: A Statement for Healthcare Professionals from the American Heart Association/ American Stroke Association. Stroke, 44, 2064-2089. http://dx.doi.org/10.1161/STR.0b013e318296aeca

[17] Mecott, G.A., Al-Mousawi, A.M., Gauglitz, G.G., Herndon, D.N. and Jeschke, M.G. (2010) The Role of Hyperglycemia in Burned Patients: Evidence-Based Studies. Shock, 33, 5-13. http://dx.doi.org/10.1097/SHK.0b013e3181af0494

[18] Garner, J.S., Jarvis, W.R., Emori, T.G., Horan, T.C. and Hughes, J.M. (1998) CDC Definitions for Nosocomial Infections. American Journal of Infection Control, 16, 128-140. http://dx.doi.org/10.1016/0196-6553(88)90053-3

[19] Hu, G.C., Hsieh, S.F., Chen, Y.M., Hsu, H., Hu, Y. and Chien, K. (2012) Relationship of Initial Glucose Level and All-Cause Death in Patients with Ischaemic Stroke: The Roles of Diabetes Mellitus and Glycated Hemoglobin Level. European Journal of Neurology, 19, 884-891. http://dx.doi.org/10.1111/j.1468-1331.2011.03647.x

[20] Nardi, K., Milia, P., Eusebi, P., Paciaroni, M., Caso, V. and Agnelli, G. (2012) Predictive Value of Admission Blood Glucose Level on Short-Term Mortality in Acute Cerebral Ischemia. Journal of Diabetes and Its Complications, 26, 70-76.

[21] Shimoyama, T., Kimura, K., Uemura, J., Saji, N. and Shibazaki, K. (2014) Elevated Glucose Level Adversely Affects Infarct volume Growth and Neurological Deterioration in Non-Diabetic Stroke Patients, but Not Diabetic Stroke Patients. European Journal of Neurology, 21, 402-410. http://dx.doi.org/10.1111/ene.12280

[22] Woo, J., Lam, C.W.K., Kay, R., Wong, A.H.Y., Teoh, R. and Nicholls, M.G. (1990) The Influence of Hyperglycemia and Diabetes Mellituson Immediate and 3-Month Morbidity and Mortality after Acute Stroke. Archives of Neurology, 47, 1174-1177. http://dx.doi.org/10.1001/archneur.1990.00530110028011

[23] Ji, R., Wang, D., Shen, H., Pan, Y., Liu, G., Wang, P., Wang, Y., Li, H., Wang, Y., and China National Stroke Registry (CNSR) Investigators (2013) Interrelationship among Common Medical Complications after Acute Stroke: Pneumonia Plays an Important Role. Stroke, 44, 3436-3444. http://dx.doi.org/10.1161/STROKEAHA.113.001931

[24] Kwan, J., Pickering, R.M., Kunkel, D., Fitton, C., Jenkinson, D., Perry, V.H., Ashburn, A.M., and Stroke Association Rehabilitation Research Centre (2013) Impact of Stroke-Associated Infection on Long-Term Survival: A Cohort Study. Journal of Neurology, Neurosurgery Psychiatry, 84, 297-304. http://dx.doi.org/10.1136/jnnp-2012-302552

[25] Butler, S.O., Btaiche, I.F. and Alaniz, C. (2005) Relationship between Hyperglycemia and Infection in Critically Ill Patients. Pharmacotherapy. The Journal of Human Pharmacology and Drug, 25, 963-976. http://dx.doi.org/10.1592/phco.2005.25.7.963

[26] Kavanagh, B.P. and McCowen, K.C. (2010) Clinical Practice. Glycemic Control in the ICU. The New England Journal of Medicine, 363, 2540-2546. http://dx.doi.org/10.1056/NEJMcp1001115 
[27] Delamaire, M., Maugendre, D., Moreno, M., Le Goff, M.C., Allannic, H. and Genetet, B. (1997) Impaired Leucocyte Functions in Diabetic Patients. Diabetic Medicine, 14, 29-34. http://dx.doi.org/10.1002/(SICI)1096-9136(199701)14:1<29::AID-DIA300>3.0.CO;2-V

[28] Matchar, D.B., Divine, G.W., Heyman, A. and Feussner, J.R. (1992) The Influence of Hyperglycemia on Outcome of Cerebral Infarction. Annals of Internal Medicine, 117, 449-456. http://dx.doi.org/10.7326/0003-4819-117-6-449

[29] Sacco, R.L., Shi, T., Zamanillo, M.C. and Kargman, D.E. (1994) Predictors of Mortality and Recurrence after Hospitalized Cerebral Infarction in an Urban Community: The Northern Manhattan Stroke Study. Neurology, 44, 626-634. http://dx.doi.org/10.1212/WNL.44.4.626

[30] Lee, T.-H., Ryu, S.-J. and Chen, S.-T. (1991) The Prognostic Value of Blood Glucose in Patients with Acute Stroke. Journal of the Formosan Medical Association, 90, 456-470.

[31] Stig-Jørgensen, H., Nakayama, H., Raaschou, H.O. and Olsen, T.S. (1994) Stroke in Patients with Diabetes: The Copenhagen Stroke Study. Stroke, 25, 1977-1984. http://dx.doi.org/10.1161/01.STR.25.10.1977

[32] National Institute for Health and Clinical Excellence (2014) Stroke: The Diagnosis and Initial Management of Acute Stroke and Transient Ischemic Attack (Clinical Guideline 68). www.nice.org.uk/CG68

[33] Jauch, E.C., Saver, J.L., Adams Jr., H.P., Bruno, A., Connors, J.J., Demaerschalk, B.M., et al. (2013) Guideline for the Early Management of Patients with Acute ischemic Stroke: A Guideline for Healthcare Professionals from the American Heart Association/American Stroke Association. Stroke, 44, 870-947. http://dx.doi.org/10.1161/STR.0b013e318284056a

[34] Kumar, S., Selim, M.H. and Caplan, L.R. (2010) Medical Complications after Stroke. Lancet Neurology, 9, $105-118$. http://dx.doi.org/10.1016/S1474-4422(09)70266-2

[35] Ji, R., Shen, H.P., Pan, Y.S., Wang, P.L., Liu, G.F., Wang, Y.L., et al. (2013) Novel Risk Score to Predict Pneumonia after Acute Ischemic Stroke. Stroke, 44, 1303-1309. http://dx.doi.org/10.1161/strokeaha.111.000598 